\title{
Parallel Determination of NeuroD1, Chromogranin-A, KI67 and Androgen Receptor Expression in Surgically Treated Prostate Cancers
}

\author{
L. Cindolo, M. Cantile, R. Franco, P. Chiodini, G. Schiavo, I. Forte, I. Zlobec, L. Salzano, \\ G. Botti, S. Gidaro, L. Terracciano, C. Cillo
}

Department of Urology, S. Pio da Pietrelcina Hospital, Vasto, Italy (LC), Department of Clinical and Experimental Medicine (MC, CC), Federico II University, Naples, Italy, Surgical Pathology (RF, IF), G. Pascale National Cancer Institute, Naples, Italy, Department of Medicine and Public Health (PC), Second University, Naples, Italy, Institute of Pathology (GS, IZ, LT, CC), University of Basel, Basel, Switzerland, Department of Urology (LS), G. Rummo Hospital, Benevento, Italy, Department of Surgical and Experimental Sciences (SG), Chieti-Pescara University, Chieti, Italy

\begin{abstract}
Purpose: Neuroendocrine differentiation is a hallmark of prostate cancer. The aim of our study was the detection of the parallel expression of neuroendocrine related markers using a prostate tissue microarray (TMA).

Materials and Methods: Our study was aimed at detecting the parallel expression of NeuroD1, Chromogranin-A (ChrA), Androgen Receptor (AR) and Ki-67 by immunohistochemistry on prostate cancer tissue microarray. The data was analyzed using SAS version 8.2 (SAS Inc, Cary, NC). The relationships between NeuroD1, ChrA and AR expressions and patients' characteristics were investigated by multivariate logistic regression analysis. Progression and Overall Survival (OS) distributions were calculated using Kaplan-Meier method.

Results: Tissue reactivity for NeuroD1, ChrA and AR concerned 73\%, 49\% and $77 \%$ of the available cases, respectively. Regarding overall survival, there were 87 deaths and 295 patients alive/censored (6 years of median follow-up). Seventyseven disease progressions occurred at the median follow-up 5.4y. A significant correlation between NeuroD1, ChrA and AR expression was observed ( $p<0.001$ and $p<0.03$, respectively). Additionally, ChrA was strongly associated in multivariate analysis to Gleason score and Ki67 expression ( $p<0.009$ and $p<0.0052$, respectively). Survival analysis showed no association between markers neither for overall nor for cancer-specific survival.

Conclusions: The results highlight that NeuroD1, Chromogranin-A and Androgen Receptor are strongly associated, however their expression does not correlate with overall survival or disease progression.
\end{abstract}

Key words: prostatic neoplasms; neuroendocrine cells; neuroD1 protein; ki-67 antigen; chromogranin A, receptors, androgen prognosis

Int Braz J Urol. 2011; 37: 57-66

\section{INTRODUCTION}

Prostate cancer (PCa) is the most frequent cancer in Western countries and the second leading cause of cancer related deaths in men $(1,2)$. The clinical course of this cancer is often unfavorable due to the shift from androgen dependent status to hormone refractoriness. The change in clinical course correlates 
with a strong increase in biological aggressiveness and a significant decrease in survival (3). Only a few studies on docetaxel-based chemotherapy have reported results in terms of survival, pain control, quality of life and progression in patients with metastatic castration-resistant prostate cancer (CRPC) $(4,5)$, albeit the risk of cytotoxic chemotherapy should be individually weighted.

In recent years, the presence of neuroendocrine differentiation (NED) features has been reported as a variable associated with the development of the CRPC $(6,7)$ during the natural history of this PCa. In general, pure neuroendocrine (NE) tumor cells do not express androgen receptors (AR), are resistant to androgen deprivation therapy and do not proliferate in response to androgens (8). Autocrine-paracrine epithelial interactions and/or transdifferentiation are the mechanisms through which NE cells act in $\mathrm{PCa}$ homeostasis (9).

The early detection of NE activity in prostate adenocarcinoma could suggest or anticipate an early diagnosis of hormones refractoriness behavior and thus justify changes in therapeutic approaches. Unfortunately, the diagnosis and the quantification of prostatic NE cell activity remains a problem. Chromogranin A (ChrA), consistently expressed during NE cell differentiation (8), is the most frequently used marker to detect NE differentiation in PCa patients, both at tissue and at serum level $(10,11)$. Nevertheless, differences between assays for serum ChrA provided a significant discordance rate, suggesting that the commercial kits for serum detection might elicit different information (12). Moreover, tissue ChrA lack prognostic significance in patients with bone metastatic $\mathrm{PCa}$ (13). Other NE markers (such as tissue CD56, synaptophysin) add only little information on the acquisition of NE phenotype in human prostate (14). Neuron-specific enolase (NSE) could become a valuable tumor progression marker and could serve as predictor of survival together with clinical parameters but only in advanced and hormone refractory prostate neoplasms $(15,16)$.

These evidences highlight that the identification of new diagnostic and prognostic markers is relevant for the clinical management of PCa patients, especially related to neuroendocrine differentiation. Following the identification of the neurogenic char- acteristic of the 2q31-33 genome region (HOX D locus) which houses genes involved with epithelialneuronal cell conversion (17), we investigated the role of NeuroD1 in normal and neoplastic human prostates. We have previously reported that NeuroD1 tissue reactivity correlates with the indicators of malignancy in moderately to poorly differentiated $\mathrm{PCa}$ and it could be involved in the pathophysiology of PCa neuroendocrine differentiation (14). Here we report on an immunohistochemical analysis using a tissue micro array (TMA) containing a high number of different naive prostate cancer specimens, in order to verify the prognostic relevance of NeuroD1 together with ChrA, AR and Ki67 tissue reactivity and their correlations.

\section{MATERIALS AND METHODS}

A total of 732 patients (members of the Kaiser Foundation Health Plan) treated for clinically localized PCa by radical prostatectomy or transurethral resection (TURP) (incidental diagnosis) at one of two Kaiser Hospitals in Portland (OR, USA) between 1971 and 1996, were retrospectively evaluated. The full study protocol, including access to the slides and blocks, was reviewed and approved by the Committee for the Protection of Human Subjects of Kaiser Permanente, Portland, OR. All patient identifiers were removed and replaced by unique study numbers, linked to the original identifiers by a single file kept under high security. Medical records for the entire cohort were abstracted at one time, 1999-2001, to assure uniform criteria for diagnosis, progression, and staging.

Selection of the specimens, classification, as well as patient management and follow-up have extensively been described elsewhere (18). Before 1992 (pre-PSA era), progression was defined clinically based on the results of bone scans, chest x-rays, and/or digital rectal examination. After 1992, progression was defined by increasing PSA serum concentrations in serial determinations following a postoperative PSA nadir value (18). Patients with $\mathrm{N}+$ or $\mathrm{M}+$ disease at the diagnosis or treated by neoadjuvant or postoperative hormonal or chemotherapy have been excluded. 
Benign prostatic hyperplasia (BPH), as control, was also evaluated in 89 specimens (not included in the analysis).

\section{Tissue Microarray Design}

The prostate TMA was constructed as previously described $(18,19)$. Briefly, one core tissuebiopsy (diameter $0.6 \mathrm{~mm}$ ) was taken from the least differentiated region of individual paraffin-embedded prostate tumors (donor blocks) and precisely arrayed into a new recipient paraffin block $(35-20 \mathrm{~mm})$ with a custom-built precision instrument (Beecher Instruments, Silver Spring, MD). The core-tissue biopsies were put into one of the two recipient blocks that defined one replicate TMA. Six replicate TMAs containing the identical set of tumors were constructed. After the block construction, $5 \mathrm{~mm}$ sections were cut using a microtome. Originally, 732 donor tissue blocks were available for the construction of this TMA. Specimens from 74 tumors could not be included in the study because of incomplete follow-up data, lack of tumor in the arrayed sample (sampling error), damaged tissue (heat or crush artifacts), or a total lack of tissue at some array positions ('empty spots'). The number of patients varies between the individual marker analyses because of variability in the number of interpretable specimens on consecutive sections.

The presence of tumor tissue on the arrayed samples was verified on a hematoxylin-eosin-stained section. All data in this study are based upon the analysis of $658 \mathrm{PCa}$ specimens.

\section{Immunohistochemistry}

Sections $(4 \mu \mathrm{m})$ of TMA blocks were transferred to an adhesive-coated slide system (Instrumedics Inc, Hackensack, NJ, USA). After incubation, immunodetection was performed following a standard avidin-biotin complex method (LSAB-DAKO; Glostrup, Denmark, and DAB; Vector Laboratories, Burlingame, CA,). The slides were immunoassayed for neuroD1 (sc-20805, 1:150; Santa Cruz Biotechnology, Santa Cruz, CA.), Ki-67 (MIB1, 1:800; Dako, Glostrup, Denmark), chromogranin A (DAK-A3,
1:100; Dako, Milan, Italy) and androgen receptor (clone AR 441 1:300 DAKO, Glostrup, Denmark).

Stained TMA sections were evaluated by pathologists using uniform criteria. In particular, single markers expression was recorded as negative/positive, considering expression in normal versus neoplastic, being the discrepancies resolved in a reviewed joint analysis.

The fraction of immunohistochemically positive cells per punch was evaluated. NeuroD1 was classified as $0 \%, 1-50 \%,>50 \%$. Chromogranin A was classified as $0-4 \%, 5-9 \%, \geq 10 \%$. For Ki67 and androgen receptor, only nuclear staining was considered. AR was classified as $0-10 \%, 11-50 \%,>50 \%$; whereas Ki67 was visually scored and stratified into two groups (low $\leq 10 \%$; high $>10 \%$ ) (18). The cut-off values used in the analyses have been selected on the bases of the best possible discriminatory effect.

\section{Statistical Analysis}

The data was analyzed using SAS version 8.2 (SAS Inc, Cary, NC). A two-tailed P value $<0.05$ was considered significant. Continuous variables were expressed as mean and Standard Deviation and compared with ANOVA. Categorical variables were expressed as a number or a percentage and compared by using Fisher's exact test. The relationships between NeuroD1, ChrA and AR expressions and patients' characteristics were investigated by multivariate logistic regression analysis. Progression and Overall Survival (OS) distributions were calculated using the Kaplan-Meier method.

\section{RESULTS}

The main clinical-pathological characteristics of the biopsies are listed in Table-1. Follow-up data for progression (median 5.4, range 0.5-20 years) were available in 631 cases. For the overall survival were useful data from 623 patients (median 6, range 2-20 years). Gleason score was assessed for all the $\mathrm{PCa}$ specimens on TMA (658 punches) and classified as well, moderately, or poorly differentiated (Gleason score $<7,7,>7$, respectively). The Gleason score and 
Table 1 - Main clinical and pathological findings of 658 patients.

\begin{tabular}{lc}
\hline Characteristics & $\mathbf{N}(\%)^{*}$ \\
\hline Median age (range), years & $65(45-92)$ \\
Surgery & \\
RP & $589(89)$ \\
TURP & $71(11)$ \\
Gleason score & \\
$<7$ & $378(57.5)$ \\
7 & $224(34.0)$ \\
$>7$ & $56(8.5)$ \\
Stage & \\
pT2 & $467(71.0)$ \\
pT3 & $105(16.0)$ \\
pT4 & $27(4.1)$ \\
pTx & $59(9.0)$ \\
High Grade PIN & $25(3.8)$ \\
Perineural invasion & $270(41.0)$ \\
Seminal vesicles invasion & $35(5.3)$ \\
Urethral invasion & $42(6.4)$ \\
NeuroD1 & \\
Absent & $107(26.2)$ \\
Intermediate & $130(31.8)$ \\
High & $172(42.1)$ \\
ChrA & \\
Low & $358(57.0)$ \\
Intermediate & $165(26.3)$ \\
High & $105(16.7)$ \\
Low & \\
Intermediate & $131(38.1)$ \\
High & $100(26.8)$ \\
High & \\
\hline
\end{tabular}

*tables entries are absolute numbers and percentages, but for age. $R P=$ Radical Prostatectomy; TURP = Transurethral resection; $P I N=$ Prostatic intraepithelial neoplasia $;$ ChrA = chromogranin$A ; A R=$ androgen receptor $;$ Ki67 $=$ Ki67 label index.

pathologic stage were highly predictive for progression $(\mathrm{p}<0.0001)$ and overall survival $(\mathrm{p}<0.0001)$.

\section{Immunohistochemistry}

A total of 409 PCa punches were available to detect for NeuroD1 protein expression. Among these, $302(73 \%)$ showed a NeuroD1 positive cytoplasmic staining (Table-1). Only few cases showed a faint nuclear stain. Results according to Gleason score were reported in Table-2. NeuroD1 expression has shown significant association with $\mathrm{ChrA}(\mathrm{p}<0.001)$ and AR expression $(\mathrm{p}<0.004)($ Table-3). Only $3 / 89$ $(3 \%)$ cases of BPH showed a weak positivity. Failure of analysis occurred in 249 cases mostly for unreliability of staining or missing/damaged tissue.

Of 628 PCa punches valuable for ChrA expression, $270(43 \%)$ showed a moderately-to-high positive staining (Table-1). For ChrA, 30 cases are invaluable or missing tissue, due to technical problems. The immunohistochemical analysis revealed a cytoplasmic positivity, whereas 206 cases were completely negative. Twenty cases of BPH were focally positive. Results according to Gleason score are reported in Table-3. ChrA expression is associated with Gleason score, NeuroD1, AR and Ki67 index (p $=0.002, \mathrm{p}<0.001, \mathrm{p}=0.004$ and $\mathrm{p}<0.001$, respectively) (Tables 2 and 3 ).

The staining for the AR was available for 373 punches of PCa (Table-1), displaying predominantly a nuclear localization. We detected a low, intermediate and high AR tissue reactivity in 38\%,35\% and $27 \%$, respectively. AR expression is associated with NeuroD 1 and $\mathrm{ChrA}(\mathrm{p}=0.004$ and $\mathrm{p}=0.004$, respectively).

A high Ki67 Labelling Index (missing 121 cases) was found in $14.5 \%$ of the 537 evaluated punches and it was significantly associated with a high ChrA expression $(\mathrm{p}<0.001)($ Table- 3$)$. The univariate analysis associates $\mathrm{ChrA}$ and Ki67 with Gleason score $(p=0.002$ and $p<0.001)($ Table-2). The multivariate analysis (Table-4) further shows all markers but AR in significant test trend association with the Gleason score. Neither ChrA, nor AR and NeuroD1 positive staining were found to be associated with the presence of seminal vesicles, urethral or perineural invasion.

The Kaplan-Meier model curves showed that Gleason score (data not shown) and Ki67 level had a significant influence on survival parameters $(p<0.001)$, whereas ChrA $(p=0.7)$, AR $(p=0.8)$ 
Table 2 - Pattern of markers expression distributed according to homogeneous pathological group of patients following Gleason score*.

\begin{tabular}{|c|c|c|c|c|}
\hline Pathological Markers & Gleason $<7$ & Gleason $=7$ & Gleason $>7$ & p Value \\
\hline Neuro D1 (missing N = 249) & & & & 0.547 \\
\hline Absent & $69(28.9)$ & $31(22.6)$ & $7(21.2)$ & \\
\hline Intermediate & $77(32.2)$ & $43(31.4)$ & $10(30.3)$ & \\
\hline High & $93(38.9)$ & $63(46.0)$ & $16(48.5)$ & \\
\hline ChrA (missing $\mathrm{N}=30$ ) & & & & 0.002 \\
\hline Low & $216(59.5)$ & $126(58.3)$ & $16(32.7)$ & \\
\hline Intermediate & $97(26.7)$ & $51(23.6)$ & $17(34.7)$ & \\
\hline High & $50(13.8)$ & $39(18.1)$ & $16(32.7)$ & \\
\hline AR (missing $\mathrm{N}=285$ ) & & & & 0.142 \\
\hline Low & $79(36.1)$ & $54(43.5)$ & $9(30.0)$ & \\
\hline Intermediate & $78(35.6)$ & $37(29.8)$ & $16(53.3)$ & \\
\hline High & $62(28.3)$ & $33(26.6)$ & $5(16.7)$ & \\
\hline Ki67 (missing $N=121)$ & & & & $<0.001$ \\
\hline Low & $282(91.6)$ & $147(80.3)$ & $30(65.2)$ & \\
\hline High & $26(8.4)$ & $36(19.7)$ & $16(34.8)$ & \\
\hline
\end{tabular}

* tables entries are absolute numbers and percentages. ChrA = chromogranin-A; AR = androgen receptor; Ki67 = Ki67 label index.

Table 3 - Spearman's correlation matrix of marker tissue reactivity*.

\begin{tabular}{lcccc}
\hline & NeuroD1 & ChrA & AR & Ki67 \\
\hline NeuroD1 & - & $0.187(<0.001)$ & $0.189(0.004)$ & $0.013(0.806)$ \\
ChrA & - & - & $0.151(0.004)$ & $0.164(<0.001)$ \\
AR & - & - & - & $0.091(0.115)$ \\
Ki67 & - & - & - & - \\
\hline
\end{tabular}

$*$ tables entries are correlation coefficients and $p$ Values. ChrA $=$ chromogranin-A; AR $=$ androgen receptor; Ki6 $7=$ Ki67 label index.

and NeuroD1 $(\mathrm{p}=0.7)$ did not show any significant influence on progression-free (Figure-1) and overall survival (data not shown).

\section{COMMENTS}

Although several immunohistochemical studies revealed the presence of NE cells in almost all PCa
(20), their prognostic relevance remain controversial (21). The NED (mainly identified by tissue ChrA positive staining) seems to be useful as predictor for biochemical failure after radical prostatectomy in clinically localized PCa (21-23) and in low Gleason score $\mathrm{PCa}$ (23). As far as NE activity is concerned it will be difficult to detect as the knowledge of NED pathophysiology remains obscure, prompting the search for new biomarkers (14). Therefore, we previ- 
Table 4 - Multivariate analysis of association with markers distributed according to homogeneous pathological groups*.

\begin{tabular}{lcccc}
\hline & NeuroD1 & ChrA & AR & Ki67 \\
\hline $\begin{array}{l}\text { Age, year } \\
\text { Gleason score } \\
\quad \text { > }\end{array}$ & $0.99(0.96-1.01)$ & $0.99(0.97-1.01)$ & $0.99(0.97-1.02)$ & $1.00(0.96-1.03)$ \\
$\quad 7$ & & & & \\
$\quad>7$ & $1.44(0.96-2.16)$ & $1.06(0.75-1.49)$ & $0.77(0.50-1.17)$ & $2.53(1.44-4.45)$ \\
Stage & $1.72(0.82-3.61)$ & $2.88(1.59-5.22)$ & $0.92(0.45-1.91)$ & $4.86(2.21-10.7)$ \\
$\quad$ pT2 (reference) & & & & \\
$\quad$ pT3 & $0.99(0.57-1.73)$ & $1.02(0.67-1.56)$ & $1.03(0.62-1.73)$ & $0.87(0.43-1.73)$ \\
$\quad$ pT4 & $0.70(0.28-1.75)$ & $1.14(0.52-2.54)$ & $0.93(0.34-2.53)$ & $1.76(0.67-4.68)$ \\
pTx & $0.91(0.47-1.76)$ & $0.71(0.38-1.32)$ & $0.94(0.48-1.87)$ & $2.24(0.95-5.27)$ \\
Perineural invasion & & & & \\
$\quad$ Yes vs No & $0.87(0.59-1.27)$ & $1.23(0.89-1.69)$ & $1.19(0.80-1.77)$ & $1.49(0.89-2.51)$ \\
\hline
\end{tabular}

Tables entries are OR and 95\% confidence intervals. ${ }^{\wedge} p$ for trend test is $<0.05$ NeuroD1, ChrA and Ki67. ChrA $=$ chromogranin-A; $A R=$ androgen receptor; Ki67=Ki67 label index.

ously investigated the effects of cAMP on epithelial prostate cancer cell lines detecting a significant variation of HOX-D gene expression and identifying the upstream area of the HOX-D locus on chromosome 2q31-33 as potentially involved in a neurogenic program connected to NED (17). Among the genes located in this genomic area, NeuroD1 expression has been related to $\mathrm{PCa}$ (14). New evidences have further stressed the use of pro-neural transcription factors, including NeuroD1, as cancer biomarkers (24), suggesting that the aberrant initiation of differentiation programs may confer a selective advantage. The observation that in different $\mathrm{PCa}$ models (human derived neoplastic cell-lines, transgenic mouse tumors and patient samples) the hallmarks of neural transdifferentiation along the progression to metastatic disease were associated with changes in the expression of activator-type beta-Helix-Loop-Helix transcription factors including Hes6 and Ascl1 (24) strongly corroborates our findings. The activation of pro-neural transcription factors may well be a crucial step in $\mathrm{PCa}$ progression even in a naïve prostate cancer. Through the use of TMA methodology, we have compared different NE markers in patients who underwent radical prostatectomy for surgically treated naïve $\mathrm{PCa}$. This immunohistochemical assay (IHC) showed a very low expression of NE markers in BPH (data not shown), as previously reported (14). On the other hand, in PCa we found a higher prevalence of NeuroD1 (73\% of the cases), Ki-67 (85\%) and AR (62\%) over ChrA expression (42\%), respectively. Herein, we showed that all the markers in our study are mutually and strongly associated (Tables 2 and 3 ).

The well-documented correlations $(18,23,25,26)$ between the Ki-67 expression and the aggressive features of $\mathrm{PCa}$ were confirmed here by the demonstration of its significant association with Gleason score, ChrA expression and survival. On the other hand, the absence of correlation with the NeuroD1 and AR (Figure-2) could be explained by the fact that Ki-67 is only a marker of proliferation, whereas NeuroD1 and AR are implicated into the neuroendocrine differentiation pathway $(9,14,27,28)$.

The evidence of significant associations between ChrA, NeuroD1 and AR probably suggests that their expression is not only correlated, but also that the biological significance remains rather obscure. We can speculate about the functional rela- 
NeuroD1

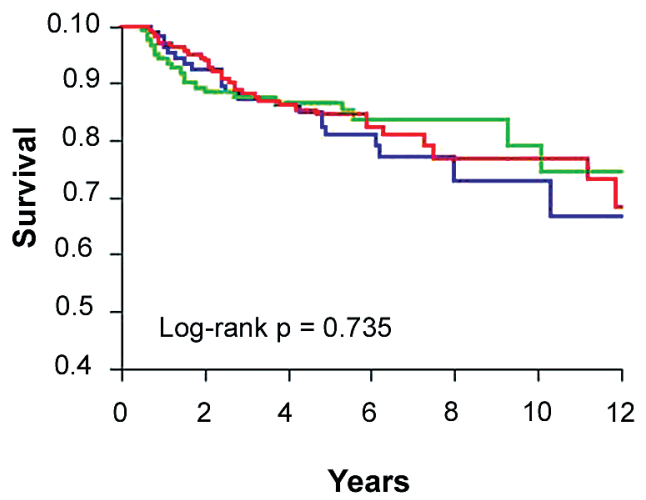

AR

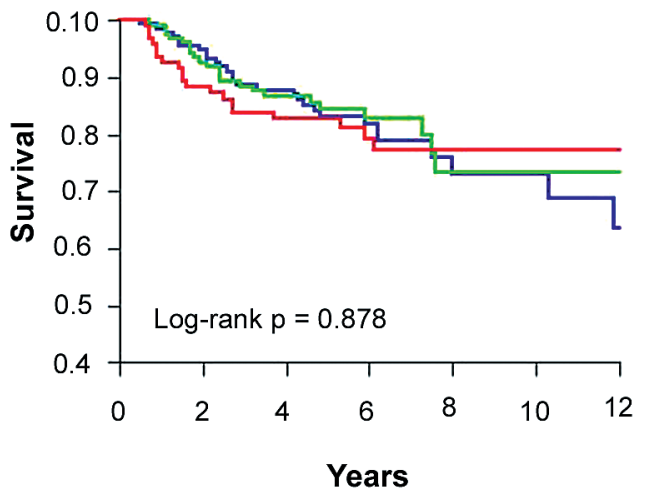

ChrA

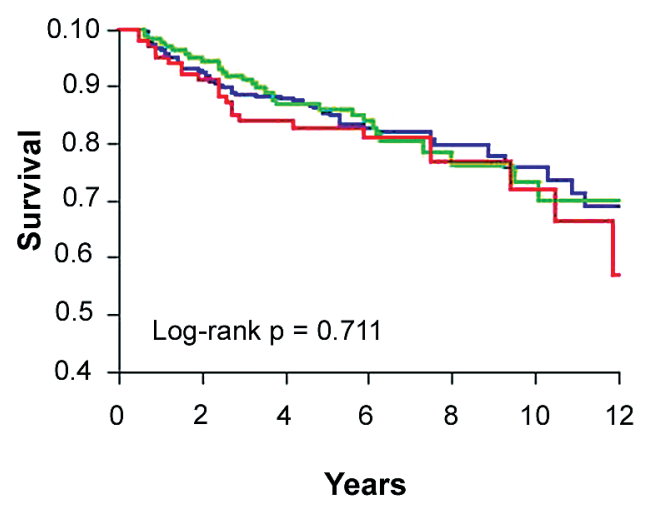

Ki67

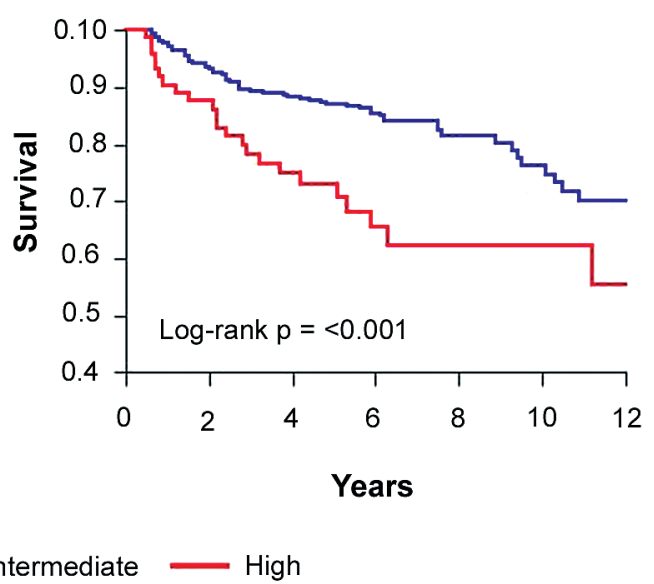

Figure 1 - Progression free survival according to the markers tissue reactivity. ChrA = chromogranin-A; AR = androgen receptor; Ki67 = Ki67 label index. In the first panel, the blue line is referred to the group without NeuroD1 tissue reactivity indicated as "low".

tionships in induction or sustain of a neuroendocrine activity or NED in PCa. In the low-grade (Gleason score $<7$ ) group NeuroD1 and ChrA were detected in 71.1 and $40.5 \%$ of the cases, respectively. In our opinion this finding is interesting and suggests that NeuroD1 could be activated in prostate tumorigenesis and that it probably is a more accurate marker of transdifferentiated cells or cells predisposed to an early NED.

Further experiments are needed to demonstrate that for the early detection of NE activity an integrated diagnostic panel (e.g. Dopa-Decarboxylase,
a-methylacyl-CoA racemase, IL-8 receptors) should be proposed (9).

A limitation of our study concerns the cut-off values used in the analyses, selected on the bases of the best possible discriminatory effect. This approach may predispose to detect false positive results. However, as Figure-1 indicates, only Ki67 robustly emerged as prognostic variable between the markers tested for prognostic implication. A clear finding of the study is the easy identification of high- and low-progression risk PCa patients, with the majority of patients belonging to the intermediate group by all the markers. The 

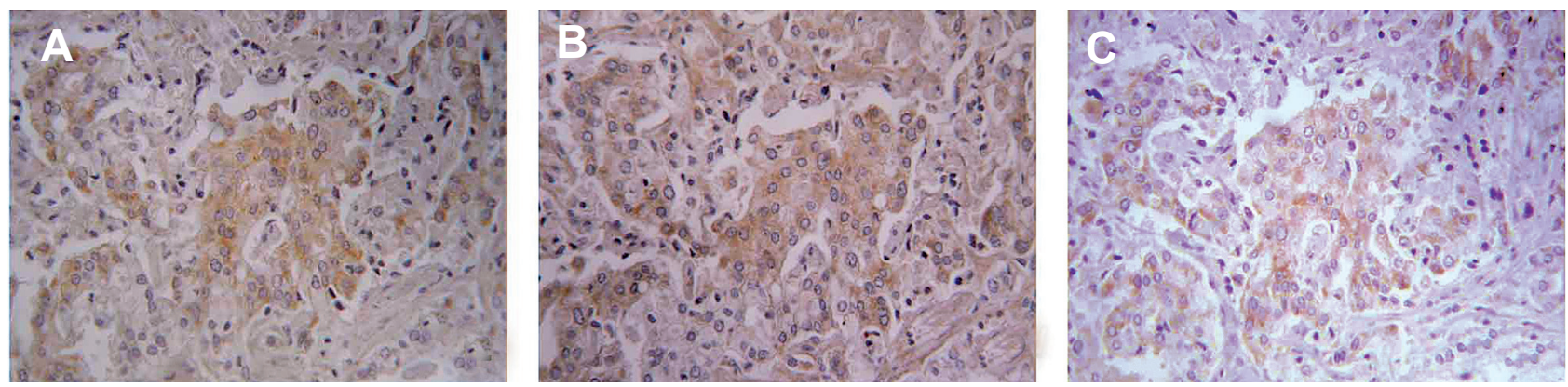

Figure 2 - Sequential slides of a same Gleason-4 prostate cancer, showing high chromogranin-A (A) and Neuro D1 (B) positivity with absent expression of androgen receptor (C). Immunohistochemical staining, X40.

intermediate group includes a significant fraction of patients who experience progression of disease, urging for additional markers. Furthermore, we have used an historical (1971-1996) series of surgically treated patients (members of the Kaiser Foundation Health Plan) for the evaluation of the prognostic significance and the internal relationships of the markers. Thus, the likelihood of biases due to patient selection, surgical management, follow-up data and tissue quality is not negligible. On the other hand, the long median followup time (almost 6 years with the longest follow-up time being over 12 years) is an interesting argument suggesting that $\mathrm{PCa}$ cells may remain dormant for long periods of time (PCa progression can also take place 10 years after prostatectomy). Moreover, data concerning the kind of progression detection (by the use of the preoperative and during the follow-up PSA values or traditional imaging test) are lacking, hindering any possible inference relationship between kind of progression, PSA, NE markers and prognosis.

\section{CONCLUSIONS}

Our study highlights the utility of TMAs to efficiently evaluate candidate prognostic markers in PCa. While some results confirm previous findings, for the first time, to our knowledge, ChrA, AR and NeuroD1 were evaluated together on a prostate TMA. The lack of association between the ChrA, AR and NeuroD1 tissue reactivity and survival suggest that these markers cannot be considered prognostic marker in patients surgically treated for
PCa. Nevertheless, a better identification of such neuroendocrine differentiation could advise about a better response rate after carboplatin-etoposide regimen chemotherapy (29).

Also, the highest reactivity of NeuroD1 over ChrA suggests its possible use, for example, as a target for antisense oligonucletide therapy (30).

\section{CONFLICT OF INTEREST}

None declared.

\section{REFERENCES}

1. Prostate Cancer Incidence and Mortality Worldwide in 2008: Globocan 2002. Available at http://globocan. iarc.fr/factsheets/cancers/prostate.asp Last access 11 Nov. 2010.

2. Altekruse SF, Kosary CL, Krapcho M, Neyman N, Aminou R, Waldron W, et al.: Edwards BK (eds). SEER Cancer Statistics Review, 1975-2007, National Cancer Institute. Bethesda, MD. Available at http:// seer.cancer.gov/csr/1975_2007/, based on November 2009 SEER data submission, posted to the SEER web site, 2010.

3. Schröder FH: Progress in understanding androgenindependent prostate cancer (AIPC): a review of potential endocrine-mediated mechanisms. Eur Urol. 2008; 53: 1129-37.

4. Tannock IF, de Wit R, Berry WR, Horti J, Pluzanska A, Chi KN, et al.: Docetaxel plus prednisone or mitoxantrone plus prednisone for advanced prostate cancer. N Engl J Med. 2004; 351: 1502-12. 
5. Petrylak DP, Tangen CM, Hussain MH, Lara PN Jr, Jones JA, Taplin ME, et al.: Docetaxel and estramustine compared with mitoxantrone and prednisone for advanced refractory prostate cancer. N Engl J Med. 2004; 351: 1513-20.

6. Jiborn T, Bjartell A, Abrahamsson PA: Neuroendocrine differentiation in prostatic carcinoma during hormonal treatment. Urology. 1998; 51: 585-9.

7. Hirano D, Okada Y, Minei S, Takimoto Y, Nemoto $\mathrm{N}$ : Neuroendocrine differentiation in hormone refractory prostate cancer following androgen deprivation therapy. Eur Urol. 2004; 45: 586-92; discussion 592.

8. Kokubo H, Yamada Y, Nishio Y, Fukatsu H, Honda N, Nakagawa A, et al.: Immunohistochemical study of chromogranin A in Stage D2 prostate cancer. Urology. 2005; 66: 135-40.

9. Cindolo L, Cantile M, Vacherot F, Terry S, de la Taille A: Neuroendocrine differentiation in prostate cancer: from lab to bedside. Urol Int. 2007; 79: 287-96.

10. Vashchenko N, Abrahamsson PA: Neuroendocrine differentiation in prostate cancer: implications for new treatment modalities. Eur Urol. 2005; 47: 147-55.

11. Sciarra A, Gentile V, Monti S, Dattilo C, Gomez AA, Salciccia S, et al.: Comparison of chromogranin A, insulin-like growth factor 1 and prostate-specific antigen serum markers in prostate adenocarcinoma and benign prostatic hyperplasia. Urol Int. 2008; 80: 68-73.

12. Zitella A, Berruti A, Destefanis P, Mengozzi G, Torta $\mathrm{M}$, Ceruti C, et al.: Comparison between two commercially available chromogranin A assays in detecting neuroendocrine differentiation in prostate cancer and benign prostate hyperplasia. Clin Chim Acta. 2007; 377: 103-7.

13. Yamada Y, Nakamura K, Aoki S, Taki T, Matsubara $\mathrm{H}$, Sai S, et al.: Is neuroendocrine cell differentiation detected using chromogranin A from patients with bone metastatic prostate cancer a prognostic factor for outcome? Oncol Rep. 2006; 15: 1309-13.

14. Cindolo L, Franco R, Cantile M, Schiavo G, Liguori G, Chiodini P, et al.: NeuroD1 expression in human prostate cancer: can it contribute to neuroendocrine differentiation comprehension? Eur Urol. 2007; 52: 1365-73.

15. Hvamstad T, Jordal A, Hekmat N, Paus E, Fosså SD: Neuroendocrine serum tumour markers in hormone-resistant prostate cancer. Eur Urol. 2003; 44: 215-21.

16. Berruti A, Dogliotti L, Mosca A, Bellina M, Mari M, Torta $\mathrm{M}$, et al.: Circulating neuroendocrine markers in patients with prostate carcinoma. Cancer. 2000; 88: 2590-7.
17. Cantile M, Kisslinger A, Cindolo L, Schiavo G, D’Antò V, Franco R, et al.: cAMP induced modifications of HOX D gene expression in prostate cells allow the identification of a chromosomal area involved in vivo with neuroendocrine differentiation of human advanced prostate cancers. J Cell Physiol. 2005; 205: 202-10.

18. Zellweger T, Ninck C, Mirlacher M, Annefeld M, Glass AG, Gasser TC, et al.: Tissue microarray analysis reveals prognostic significance of syndecan-1 expression in prostate cancer. Prostate. 2003; 55: 20-9.

19. Kononen J, Bubendorf L, Kallioniemi A, Bärlund M, Schraml P, Leighton S, et al.: Tissue microarrays for high-throughput molecular profiling of tumor specimens. Nat Med. 1998; 4: 844-7.

20. Abrahamsson PA, Wadström LB, Alumets J, Falkmer $\mathrm{S}$, Grimelius L: Peptide-hormone- and serotonin-immunoreactive cells in normal and hyperplastic prostate glands. Pathol Res Pract. 1986; 181: 675-83.

21. Komiya A, Suzuki H, Imamoto T, Kamiya N, Nihei N, Naya Y, et al.: Neuroendocrine differentiation in the progression of prostate cancer. Int J Urol. 2009; 16: 37-44.

22. Revelos K, Petraki C, Scorilas A, Stefanakis S, Malovrouvas D, Alevizopoulos N, et al.: Correlation of androgen receptor status, neuroendocrine differentiation and angiogenesis with time-to-biochemical failure after radical prostatectomy in clinically localized prostate cancer. Anticancer Res. 2007; 27 : 3651-60.

23. May M, Siegsmund M, Hammermann F, Loy V, Gunia $\mathrm{S}$ : Prognostic significance of proliferation activity and neuroendocrine differentiation to predict treatment failure after radical prostatectomy. Scand J Urol Nephrol. 2007; 41: 375-81.

24. Vias M, Massie CE, East P, Scott H, Warren A, Zhou $\mathrm{Z}$, et al.: Pro-neural transcription factors as cancer markers. BMC Med Genomics. 2008; 1: 17.

25. Laitinen S, Martikainen PM, Tolonen T, Isola J, Tammela TL, Visakorpi T: EZH2, Ki-67 and MCM7 are prognostic markers in prostatectomy treated patients. Int J Cancer. 2008; 122: 595-602.

26. Rubio J, Ramos D, López-Guerrero JA, Iborra I, Collado A, Solsona E, et al.: Immunohistochemical expression of Ki-67 antigen, cox-2 and Bax/Bcl-2 in prostate cancer; prognostic value in biopsies and radical prostatectomy specimens. Eur Urol. 2005; 48: 745-51.

27. Zelivianski S, Verni M, Moore C, Kondrikov D, Taylor $\mathrm{R}$, Lin MF: Multipathways for transdifferentiation of human prostate cancer cells into neuroendocrine-like 
phenotype. Biochim Biophys Acta. 2001; 1539: 2843.

28. Yuan TC, Veeramani S, Lin MF: Neuroendocrine-like prostate cancer cells: neuroendocrine transdifferentiation of prostate adenocarcinoma cells. Endocr Relat Cancer. 2007; 14: 531-47.

29. Loriot Y, Massard C, Gross-Goupil M, Di Palma M, Escudier B, Bossi A, et al.: Combining carboplatin and etoposide in docetaxel-pretreated patients with castration-resistant prostate cancer: a prospective study evaluating also neuroendocrine features. Ann Oncol. 2009; 20: 703-8.

30. Hadaschik BA, Sowery RD, Gleave ME: Novel targets and approaches in advanced prostate cancer. Curr Opin Urol. 2007; 17: 182-7.

\section{Correspondence address:}

Dr. Luca Cindolo

Department of Urology

"S. Pio da Pietrelcina" Hospital

Via C. De Lellis, 1

Vasto, 66054, Italy

E-mail: lucacindolo@virgilio.it 